\title{
Usefulness of Ultrasound in Characterization of Focal Hepatic Lesions
}

\author{
Authors \\ Thahirabi .K.A ${ }^{1}$, Elizabeth Daniel ${ }^{2}$, Brahmadathan .M.N ${ }^{3}$, Raini .K.P \\ ${ }^{1}$ Associate Professor, Department of Radiodiagnosis, Medical College, Thrissur, Kerala, India \\ ${ }^{2,4}$ Assistant Professor, Department of Radiodiagnosis, Medical College, Thrissur, Kerala, India \\ ${ }^{3}$ Professor \& HOD, Department of Radiodiagnosis, Medical College, Thrissur, Kerala, India \\ Corresponding Author \\ Elizabeth Daniel \\ Assistant Professor, Department of Radiodiagnosis, Medical College, Thrissur, Kerala, India \\ Ph: 9072849574, Email: lezidan@yahoo.com
}

\begin{abstract}
Introduction: The aim of this study was to analyze the ultrasound features favoring malignancy in the evaluation of focal liver lesions, to study the imaging spectrum of such lesions, and to correlate the ultrasound findings with FNAC.

Methods: The prospective study included 48 patients who were referred to the radiology department with clinically suspected malignant hepatic pathologies who were found to have focal liver lesions by ultrasound imaging. Ultrasound guided-fine needle aspiration cytology was performed in all these 48patients and correlation of usg-fnac results made out.

Results: Out of 48 patients diagnosed by ultrasound, the most common focal liver lesions seen in our study were liver metastasis (68.8\%), HCC (12.5\%) and abscess (18.8. On FNAC (72.9\%) were metastatic lesions, HCC constituted (10.4\%) and Abscesses (16.7\%).

Conclusion: Ultrasound is a safe and effective method of detecting focal liver lesion. Its low cost, easy availability and lack of ionizing radiation and iodinated contrast media makes it most ideal for imaging the liver. It aids in defining therapeutic decision quickly and allows ultrasound guided interventions. High degree of specificity of ultrasound diagnosis in the present study confirms the value of ultrasonographic evaluation of focal liver lesions and suggests that it can be effectively used in the routine diagnostic work.

Keywords: Focal liver lesions, ultrasound, Fine needle aspiration cytology.
\end{abstract}

\section{Introduction}

Liver diseases are amongst the common causes of morbidity and mortality in India, which are encountered frequently in day-to-day practice. To establish the correct diagnosis and treatment, a precise initial diagnostic imaging modality is needed. Following history and clinical examination, ultrasonography has become one of the first and most useful methods of investigation in patients with upper abdominal pain, jaundice and mass per abdomen. It is a testament to the importance of ultrasonography that almost $25 \%$ of all imaging studies worldwide are ultrasonographic examinations. Ultrasound is widely accessible, inexpensive, non-invasive, and portable with high spatial and temporal resolution. Ultrasound is the 
first choice of investigation for screening of patients with suspected liver diseases. Focal liver lesions mainly comprise of liver abscess, cystic lesions, primary malignant neoplasms metastases, focal fatty infiltrations and hematoma. The signs and symptoms of such lesions are non-specific and biochemical tests have limitations in the diagnosis of these lesions. Real-time ultrasonography has got considerable application in diagnosis of focal liver lesions. It gives valuable information regarding other parameters such as site, size, number of lesions, nature of lesions and relation to surrounding structures. Ultrasonography has an important role in the detection and follow-up of focal liver lesions. It can be used as an imaging guide for FNAC and therapeutic drainage of abscesses. This study has been conducted to diagnose different commonly encountered types of focal liver lesions by ultrasonography as a prime imaging modality and to assess the validity of ultrasonographic diagnosis in relation to FNAC diagnosis.

\section{Aims \& Objectives}

1. To assess the role of ultrasonography as the prime diagnostic modality in the characterization of hepatic lesions.

2. To study the validity of ultrasonographic diagnosis in relation to Fine Needle aspiration Cytology (FNAC) diagnosis

3. To assess the sensitivity and specificity of ultrasonography in the evaluation of focal liver lesions

\section{Materials and Methods}

The ethics committee of our institute approved this prospective study and an informed consent was taken from all patients included in it. The prospective study included 48 patients with clinically suspected focal liver pathologies seen at Govt. Medical College, Thrissur, Kerala, India. However patients with history of trauma and those with known hepatic hemangioma, simple cyst or Storage disorders were excluded. Our study group consisted of 48 cases belonging to all age groups. All patients were referred from various departments of this hospital with clinical suspicion of hepatic malignancy .The name, age, unit, registration number and address of the patients were recorded. Clinical history of jaundice, fever and loss of weight were noted. Laboratory findings of liver function test and promthrombin time were recorded.

Conventional gray scale sonography and vascularity of lesions were performed with 2-5 MHZ frequency curvilinear transducer on a real time Doppler Ultrasound Machine, PHILIPS En Visor HD version c.1.2. All patients underwent FNAC with 20-22 G spinal needle. The examination was begun with the patient in the supine position. Scan was performed in all planes. Patient was asked to take in and hold breath or to push out the anterior abdomen for better visualization of hepatic dome. The lesions were identified, characterized and vascularity of the mass was assessed. The observations were noted in a pre-decided proforma. Those patients with sonological diagnosis of metastasis, abscess and HCC were further evaluated by USG guided FNAC. FNAC was performed after explaining the procedure to the patient and taking an informed written consent. The area was cleaned with betadine. Betadine was used as coupling agent for USG. Local anaesthesia was given only in highly apprehensive patient. The right lobe lesions and left lobe lesions usually underwent aspiration by a subcostal approach with patient in supine position. The lesions in the dome or high right lobe were approached by an intercostals approach with the patient in the left posterior oblique position and needle passed in the suspended respiration. The procedure was done using 20-22 gauge spinal needle. Free hand technique under the guidance of USG done and aspiration achieved by multiple passages through the lesion. Four wet slides were prepared and sent for cytological evaluation in the preservative of absolute alcohol. After the procedure the patients were observed and repeat USG done to look for complications. No procedure related complications were observed in any of the cases. 


\section{Results}

In our study 48 patients with clinical diagnosis of malignant hepatic lesions were studied with Doppler sonogrphy and USG guided FNAC. After sonography the hepatic lesions characterized as metastatic, HCC and abscess. Among these patients the most common symptom was loss of weight $(63 \%)$ Others were fever $(27 \%)$ and jaundice (12\%). Age ranged from 15 to 80 . Youngest patient, who had history of ovarian neoplasm, found to have a solitary metastatic hepatic lesion. Oldest was a female, presented with fever, diagnosed to have Hepaocellular carcinoma.

Based on ultrasound morphology the hepatic focal lesions were categorized into metastatic lesions $(68.8 \%)$, HCC (12.5\%) and abscess (18.8\%) ON FNAC $(72.9 \%)$ were metastatic lesions. HCC constituted (10.4\%) and Abscesses (16.7\%)

1. Metastatic lesions: Out of 33 cases 28 were multiple and 5 cases were solitary. The number lesions classified according to their echogenicity were hypoechoic lesions (12) target lesions (10) hyperechoic (8), Calcific matastasis (1), mixed lesion (1) and necrotic lesion (1). Hypoechoic halo noted in 17 cases, intralesional, peripheral vascularity noted in 8 cases. Central necrosis found in $10 \%$ of metastatic lesions and $25 \%$ of HCC in our study. Small areas of necrosis were seen in metastasis and larger areas of necrosis in HCC.

2. Hepatocellular carcinoma: The sonological diagnosis of HCC were made for the hepatic focal lesions in the setting of chronic parenchymal disease except for one case where large solitary irregular heterogen-eously hyperechoic lesion with central vascularity reported as HCC in a young male of 38 years with normal appearing liver. One case was multiple small hypoechoic lesions of varying size with irregular border and three were hyperechoic solitary lesions with central vascularity. One case showed hypoechoic halo. Of the six cases of USG diagnosed
HCC. 4 cases were proved after FNAC and serum AFP evaluation. Two irregular solitary lesions with internal vascularity turned out to be as metastatic lesion. One large irregular solitary heterogeneous lesion with central cystic area and internal debris proved as HCC on USG.

3. Hepatic abscess: There were both solitary and multiple abscesses in our study. Of the 6 cases of solitary lesions 3 cases were hypoechoic with echogenic wall and posterior shadowing. 2 were hyperechoic with ill defined border and one mixed. They were round or ovoid with a discrete, irregular, echo-poor margin. The 2 cases of multiple abscess were diffusely echogenic having acoustic enhancement and echogenic

Table 1 : USG diagnosis of cases

\begin{tabular}{|l|c|c|}
\hline Lesions & Frequency & Percent \\
\hline Metastasis & 33 & 68.8 \\
\hline Hepatocellular carcinoma & 6 & 12.5 \\
\hline Abscess & 9 & 18.8 \\
\hline Total & 48 & 100 \\
\hline
\end{tabular}

thick wall.

Table 2 : FNAC diagnosis of cases

\begin{tabular}{|l|c|c|}
\hline Lesions & Frequency & Percent \\
\hline Metastasis & 35 & 72.9 \\
\hline Hepatocellular carcinoma & 5 & 10.4 \\
\hline Abscess & 8 & 16.7 \\
\hline Total & 48 & 100 \\
\hline
\end{tabular}

\section{Discussion}

In this prospective study, 48 patients with clinical diagnosis of malignant hepatic lesions underwent USG and USG guided FNAC. The age group of the patient ranged from 15-80 years. The male female ratio was 1.6: 1 . Out of 48 cases, there were 39 cases of malignant lesions (33metastasis and 6 HCC) and 9 cases of abscesses on ultrasound.

Out of the 33 cases of sonologically diagnosed metastatic lesions, 32 cases were proved as metastases on FNAC and 1 case as HCC. The sensitivity, specificity and positive predictive value of USG in the evaluation of metastasis were calculated and they were $91.4 \%, 92.3 \%$ and $97 \%$ 


\section{JMSCR Vol||05||Issue||07||Page 25233-25237||July}

respectively. Out of the 6 cases of sonologically reported HCC. 4 cases were confirmed, as HCC. There were 2 false positive diagnoses of HCC for metastasic lesions and 1 false negative diagnosis of abscess. So the sensitivity, specificity and positive predictive value in the evaluation of HCC were $80 \%, 95.3 \%$ and $66.7 \%$ respectively.

Out of the 9 cases of sonologically diagnosed abscesses, 7 cases were abscess on FNAC. Two cases diagnosed as abscess turned out to be metastatic lesions. The sensitivity, specificity and positive predictive value in the evaluation of abscess were $87.5 \%, 95 \%$ and $77.8 \%$ respectively.

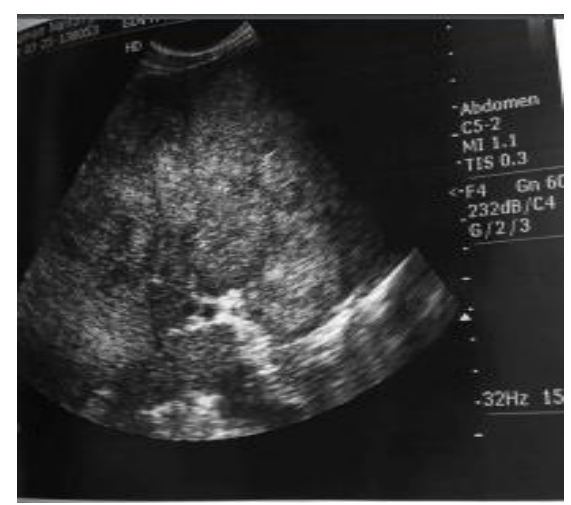

Figure 1: Hyperechoic metastatic lesions with halo

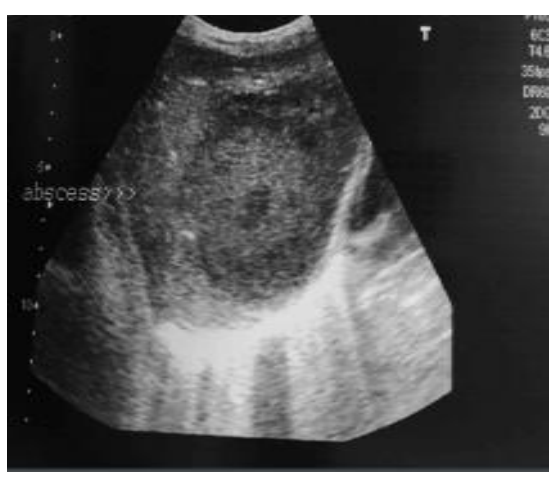

Figure 2: Hepatic abscess

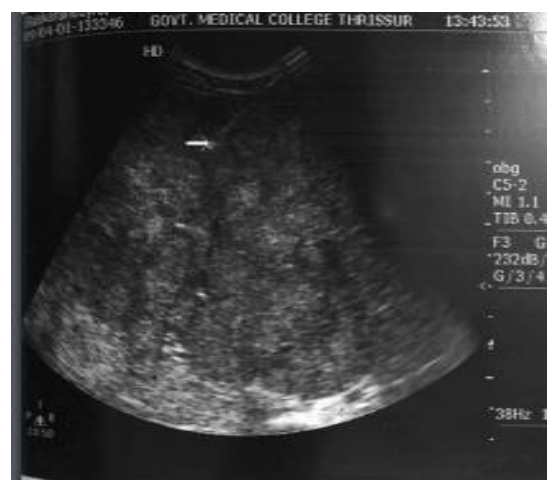

Figure 3: Guided FNAC Needle Track

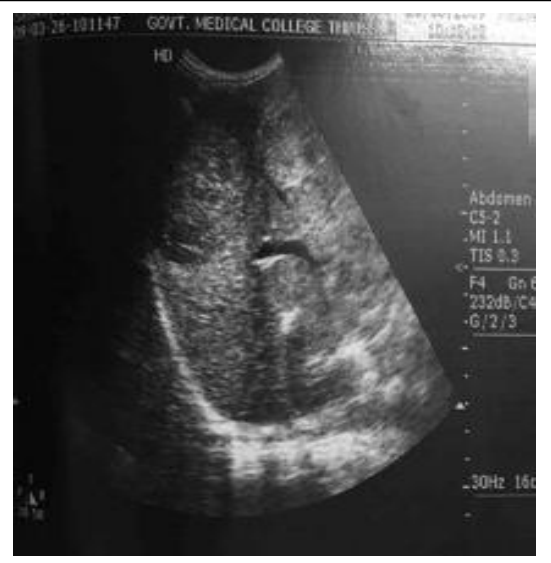

Figure 4: Hepatocellular carcinoma

A prospective study conducted in 50 patients by Nasir T A et at ${ }^{1}$ in 2001 found USG has got specificity of $83 \%$ in evaluating multiple lesions as metastasis and $90 \%$ specificity in evaluating solitary neoplastic lesions.

A retrospective study was undertaken by Hers Zeyi $\mathrm{L}$; Farinati $\mathrm{F}$ et at to evaluate the diagnostic usefulness of 244 sonographically guided fine needle aspiration biopsy in 226 patients with ultrasonologically suspected hepatic malignant lesions. The diagnostic sensitivity of this technique was $93 \%$ with $100 \%$ specificity and the correlation of $80 \%$. They reported one case of needle tract tumor seeding after biopsy in 166 cases. These results confirm the usefulness of sonographically guided fine needle aspiration biopsy in diagnosing malignant hepatic tumor. The procedure is simple, safe and free of important side effects. The authors believe that USG guided FNAC represent the first choice of invasive technique in the assessment of hepatic focal benign lesions and malignant tumors.

Sherman et al ${ }^{2}$ reported that ultrasound showed a sensitivity of $71.4 \%$ specificity $93.8 \%$ and $14 \%$ of +ve predictive value in the evaluation HCC. Chalasani et at ${ }^{3}$ compared sensitivity between US and CT and sensitivity of $59 \%$ lower than CT (91\%) Teefey et al mentioned sensitivity of US (89\%) was much higher than CT (67\%) and MRI $(56 \%)$.

Yoshida T, Matsue H, Okazakin et $\mathrm{al}^{4}$ conducted studies on ultrasonographic differentiation of HCC from metastatic liver lesions and found USG has got high specificity and sensitivity. Hepatocellular 
Carcinoma Presenting as Pyogenic Liver Abscess was reported from the Department of Surgery and Pathology, Chang Gung Memorial Hospital Chang Gung University, Taipei, Taiwan ${ }^{5}$.

\section{Conclusion}

We studied 48 patients with clinical suspicion of malignancy to evaluate the role of USG to characterize focal hepatic lesions. The results were correlated with USG guided FNAC. Ultrasound guided FNAC is a simple and safe procedure. USG has got a definitive role in the characterization of focal hepatic lesions as metastasis, Abscess and HCC. USG has got $91.4 \%$ sensitivity, $92.3 \%$ specificity and $97 \%$ positive predictive value to categorize the lesion as metastasis USG has a major role in characterization of lesion as HCC with sensitivity $80 \%$ specificity $95.3 \%$ and the positive predictive value $66.7 \%$. In the abscess cases USG has got $87.5 \%$ sensitivity, $95 \%$ specificity and $77.6 \%$ positive predictive value.

\section{References}

1. Nasir TA,Banu NA, Hussain M Begum AA Ali $\mathrm{H}$ Correlation Of Ultrasonographic finding with Ultrasound guided FNAC in liver lesion Gastroenterology 2003.

2. Sherman M,Pettekian KM ,Lee screening for HCC in chronic carriers of HCC. Gastroentrology 1996.

3. Chalassini N Horlander JC Sr Said A Kopecky KK Stockberger SMJ Screening for HCC IN Advanced Cirrhosis Tropical Gastroentrology 1989.

4. Yoshida. T Matsue $\mathrm{H}, \mathrm{O}$ Kasaki $\mathrm{N}$ et al Ultrasonographic differentiation of hepatocellular carcinoma from hepatic metstasis J Clini Ultrasound 1987.

5. Hepatocellular Carcinoma Presenting as Pyogenic Liver Abscess: Characteristics, Diagnosis, and Management From the Department of Surgery and Pathology, Chang- Gung Memorial Hospital, ChangGung University,Taipei,Taiwan Clinical Infectious Diseases 1998;6:1224-1226, 1998 by the Infectious Diseases Society of America. Arights reserved. From the Department of Surgery and Pathology, Chang- Gung Memorial Hospital, ChungGung University, Taipei, Taiwan. 